\title{
Approximate analytical solution to a time-fractional Zakharov-Kuznetsov equation
}

\author{
Zakia Hammouch*, Toufik Mekkaoui \\ Équipe E3MI, Département de Mathématiques Faculté des Sciences et Techniques, Université Moulay Ismail \\ BP 509 Boutalamine 52000 Errachidia Morocco \\ *Corresponding author E-mail: hammouch.zakia@gmail.com
}

\begin{abstract}
In this paper we present approximate analytical solution of a time-fractional Zakharov-Kuznetsov equation via the fractional iteration method. The fractional derivatives are described in the Caputo sense. The approximate results show that the fractional iteration method is a very efficient technique to handle fractional partial differential equations.
\end{abstract}

Keywords: Fractional Iteration Method (FIM); Zakharov-Kuznetsov Equation; Caputo derivative.

\section{Introduction}

Nonlinear partial differential equations of fractional order play an important role in various fields of sciences and technology, because they describe many nonlinear phenomena. Unfortunately, most of fractional differential equations (FDEs) do not have exact analytical solutions, therefore considerable heed has been focused on the approximate and numerical solutions of these equations. In recent years, many methods have been developed for constructing approximate analytical solutions such as, the Adomian decomposition method (ADM)[1], the homotopy analysis method (HAM)[1], the homotopy perturbation method (HPM)[9], the variational iteration method (VIM) [2],the generalized Taylor polynimials [16], etc . Recently, Ghorbani [3] published a very interesting work whereby the approximate analytical solution of some fractional differential equations was given using a new method called Fractional Iteration Method (FIM). It was shown that this new method is very efficient and more simple to use than both of the Adomian decomposition method and the variational iteration method; since the first method has limitation due to complicated algorithms of calculating Adomian polynomials for nonlinear fractional problems and the second one requires the identification of the Lagrange multiplier which make its applicability sometimes more difficult. More recently, Shateri and Ganji [4] employed (FIM) to solve a system of nonlinear fractional Hirota-Satsuma equations, they demonstrated the accuracy of (FIM) in few iterations. Motivated by the works [3] and [4], we aim to obtain approximate solutions of the following time-fractional Zakharov-Kuznetsov equation

$$
D_{t}^{\alpha} u+a\left(u^{p}\right)_{x}+b\left(u^{q}\right)_{x x x}+c\left(u^{r}\right)_{x y y}=0 .
$$

where $u:=u(x, y, t), \alpha$ is a parameter describing the order of the fractional derivative $(0<\alpha \leq 1), a, b$ and $c$ are arbitrary constants and $p, q$ and $r$ are integers such that $p, q, r \neq 0$.

Equation (1) describes the nonlinear development of ion-acoustic waves in a magnetized plasma under the restrictions of small wave amplitude, weak dispersion, and strong magnetic fields [5][6].

Recently Batiha [7] used VIM for seeking numerical and analytical solutions for equation (1) when $\alpha=1$, with fully nonlinear dispersion. More recently, Molliq et al [8] applied (VIM) successfully to solve the equation (1) for $(0<\alpha \leq 1)$. In [9], the (HPM) has been applied for obtaining analytical solutions to (1).

The paper is organized as follows: In section 2 we provide the reader with the essential notions and notations concerning fractional calculus which will be used throughout the paper. Section 3 outlines the basic concept of the (FIM). In section 4 , we will study a special case of (1), namely $Z K(2,2,2)$ with a specific initial condition in order 
to stress how the (FIM) is efficient and accurate to obtain approximate solutions. Finally we give some concluding remarks in the last section.

\section{Essentials of fractional calculus}

The commonly used definitions for the general fractional calculus are Riemann-Liouville and Caputo [10].

Definition 2.1 A real function $f(x), x>0$, is said to be in the space $C_{\mu}, \mu \in \mathbb{R}$ if there exits a real number $\lambda>\mu$ such that $f(x)=x^{\lambda} g(x)$, where $g(x) \in C[0, \infty)$ and it is said to be in the space $C_{\mu}^{m}$ if and only if $f^{(m)} \in C_{\mu}$ for $m \in \mathbb{N}$.

Definition 2.2 The Riemann-Liouville fractional integral operator of order $\alpha$ of a real function $f(x) \in C_{\mu}, \mu \geq-1$, is defined as

$$
J^{\alpha} f(x)=\frac{1}{\Gamma(\alpha)} \int_{0}^{x}(x-t)^{\alpha-1} f(t) d t, \quad \alpha>0, x>0 \quad \text { and } \quad J^{0} f(x)=f(x) .
$$

The the operators $J^{\alpha}$ has some proprieties, for $\alpha, \beta \geq 0, \gamma, \mu \geq-1$ :

- $J^{\alpha} J^{\beta} f(x)=J^{\alpha+\beta} f(x)$,

- $J^{\alpha} J^{\beta} f(x)=J^{\beta} J^{\alpha} f(x)$,

- $J^{\alpha} x^{\xi}=\frac{\Gamma(\xi+1)}{\Gamma(\alpha+\xi+1)} x^{\alpha+\xi}$.

Next we define the Caputo fractional derivatives $D^{\alpha}$ of a function $f(x)$ of any real number $\alpha$ such that $m-1<\alpha \leq m$, $m \in \mathbb{N}$, for $x>0$ and $f \in C_{-1}^{m}$ in the terms of $J^{\alpha}$ as

$$
D^{\alpha} f(x)=J^{m-\alpha} D^{m} f(x)=\frac{1}{\Gamma(m-\alpha)} \int_{0}^{x}(x-t)^{m-\alpha-1} f^{(m)}(t) d t
$$

and has the following proprieties for $m-1<\alpha \leq m, m \in \mathbb{N}, \mu \geq-1$ and $f \in C_{\mu}^{m}$

- $D^{\alpha} J^{\alpha} f(x)=f(x)$,

- $J^{\alpha} D^{\alpha}=f(x)-\sum_{k=0}^{m-1} f^{(k)}\left(0^{+}\right) \frac{x^{k}}{k !}$, for $x>0$.

We stress here that the fractional derivatives introduced by Caputo [11] allows traditional initial and boundary conditions to be included in the formulation of the considered problem. In this paper, the time-fractional derivative is taken as the following

$$
D_{t}^{\alpha} u(x, y, t)= \begin{cases}\frac{1}{\Gamma(m-\alpha)} \int_{0}^{t}(t-s)^{m-\alpha-1} \frac{\partial^{m} u(x, y, s)}{\partial s^{m}} d s, & \text { for } \quad m-1<\alpha \leq m \\ \frac{\partial^{m} u(x, y, t)}{\partial t^{m}} & \text { for } \quad \alpha=m \in \mathbb{N}\end{cases}
$$

\section{Description of FIM}

The fractional iteration method changes the differential equation to a recurrence sequence of functions. The limit of that sequence is considered as the solution of the partial differential equation. Now consider the partial differential equation in the following form (more general form can be considered without loss of generality):

$$
D_{t}^{\alpha}+F\left(u, u_{x}, u_{x x}, u_{x y}, u_{x y y}\right)=0
$$

subjected to the initial condition

$$
u(x, y, 0)=f(x, y) .
$$

Where $F$ is a given nonlinear function and $f$ is the initial condition. 
The main task is to find a solution $u:=u(x, y, t)$ to problem $(5),(6)$ under the form

$$
u(x, y, t)=\lim _{n \rightarrow \infty} u_{n}(x, y, t)
$$

Let $H(t) \neq 0$ denotes the so-called auxiliary function. Multiplying (5) by $H(t)$ and applying the Riemann-Liouville integral operator $J^{\alpha}$ on both sides we get

$$
J^{\alpha}\left(H(t)\left[D_{t}^{\alpha} u-F\right]\right)=0 .
$$

Let $h$ denotes the auxiliary parameter. Multiplying (8) by $h$ and adding $u$ on both sides we get

$$
u(x, y, t)=u(x, y, t)+h J^{\alpha}\left(H(t)\left[D_{t}^{\alpha} u(x, y, t)-F\left(u, u_{x}, u_{x x}, u_{x y}, u_{x y y}\right)\right]\right) .
$$

Consequently, equation (8) can be solved iteratively as follows

$$
u_{n+1}(x, y, t)=u_{n}(x, y, t)+h J^{\alpha}\left(H(t)\left[D_{t}^{\alpha} u_{n}(t)-F\left(u_{n}, u_{n x}, u_{n x x}, u_{n x y}, u_{n x y y}\right)\right]\right),
$$

where the subscript $n$ denotes the iteration order. The convergence of (10) is guaranteed by Banach's fixed point theorem [12], provided that the right hand of (10) is a contractive mapping [3]. On the other hand the convergence region and the rate of the convergence of the obtained series solutions can be controlled by the convergence parameter $h$ as it is described in the framework of the homotopy analysis method (HAM) [13]. Actually, by plotting the solution (or one of its derivatives) at a particular point with respect to the auxiliary parameter $h$ which is the so-called $h$-curve, one can get a proper value of $h$ that ensures the convergence of the obtained solution series [4]. For more details about the mathematical properties of the $h$-curve we refer the readers to a recent paper by Abbasbandy et al.[15].

\section{Application}

Consider the time-fractional $\operatorname{FZK}(2,2,2)[8]$ in the form

$$
D_{t}^{\alpha} u+\left(u^{2}\right)_{x}+\frac{1}{8}\left(u^{2}\right)_{x x x}+\frac{1}{8}\left(u^{2}\right)_{x y y}=0 .
$$

The exact solution to Eq.(11) for the non-fractional case and subject to the initial condition

$$
u(x, y, 0)=\frac{4}{3} \rho \sinh ^{2}(x+y),
$$

where $\rho$ is an arbitrary constant was given in [14] as

$$
u(x, y, t)=\frac{4}{3} \rho \sinh ^{2}(x+y-\rho t) .
$$

Applying FIM to (11) as the following :

1. First, we choose $H(x, y, t)=1$.

2. Then, we construct the iterative scheme for (11) as follows :

$$
u_{n+1}(x, y, t)=u_{n}(x, y, t)+h J^{\alpha}\left[D_{t}^{\alpha} u_{n}+\left(u_{n}^{2}\right)_{x}+\frac{1}{8}\left(u_{n}^{2}\right)_{x x x}+\frac{1}{8}\left(u_{n}^{2}\right)_{x y y}\right] .
$$

3. Starting with the initial approximation (12) we get

$$
\begin{aligned}
& u_{0}(x, y, t)=u(x, y, 0)=\frac{4}{3} \rho \sinh ^{2}(x+y), \\
& u_{1}(x, y, t)=\frac{4}{3} \rho \sinh ^{2}(x+y)+\frac{32}{9} \frac{h \sinh (x+y) \cosh (x+y)\left(10 \cosh ^{2}(x+y)-7\right) \rho^{2} t^{\alpha}}{\Gamma(1+\alpha)}, \\
& u_{2}(x, y, t)=u_{1}(x, y, t)+h J^{\alpha}\left[D_{t}^{\alpha} u_{1}+\left(u_{1}^{2}\right)_{x}+\frac{1}{8}\left(u_{1}^{2}\right)_{x x x}+\frac{1}{8}\left(u_{1}^{2}\right)_{x y y}\right],
\end{aligned}
$$


and so on. In general, by means of the h-curve parameter, it is straightforward to choose a proper value of $h$ which ensures that the solution series is convergent. This proper value of $h$ corresponds to the curve segment nearly parallel to the horizontal axis. For this study we take $h=-0.4$ (see Figure.1).

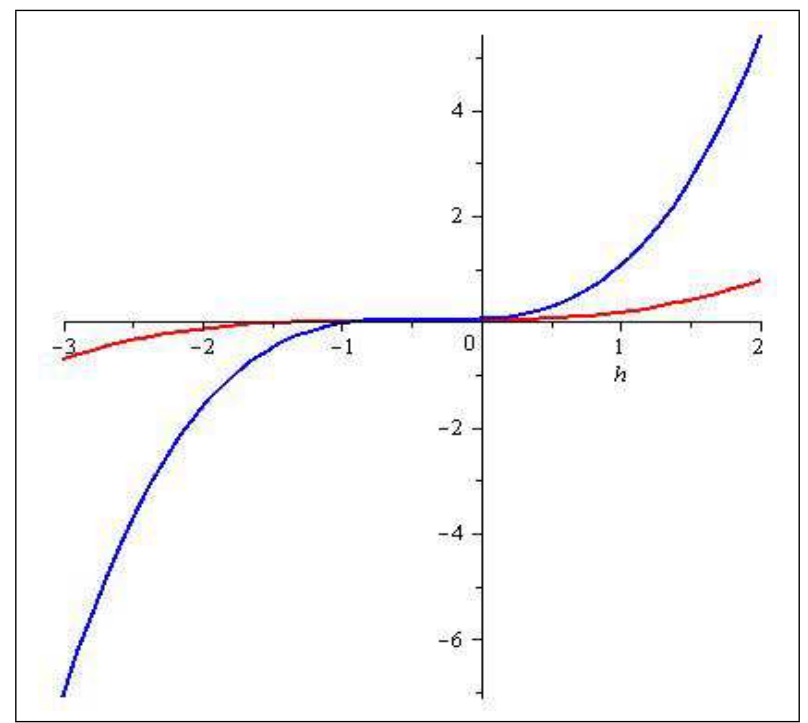

Figure 1: $h$-curves of $u(x, y, t)$ for $\alpha=1, \rho=0.001$ at a point $(0.5,0.5,2)$.

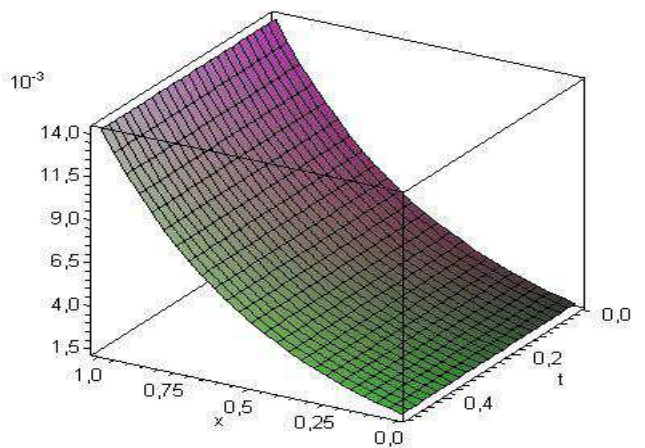

(a)

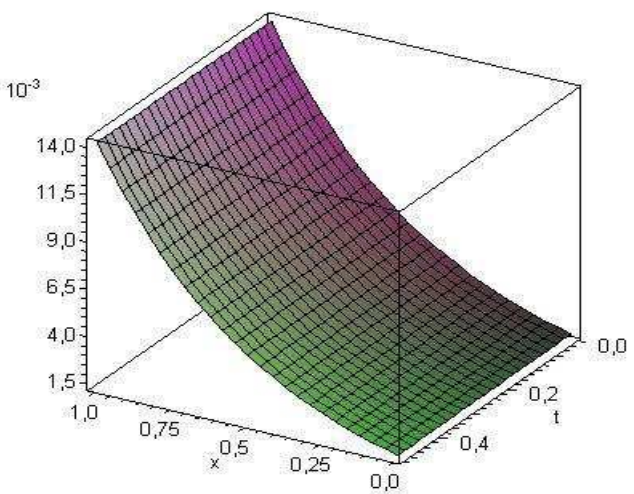

(b)

Figure 2: Profiles of $u(x, y, t)$ when $\alpha=1, \rho=0.001$ and $y=0.9:$ (a) Exact solution of (11) and (b) Approximate solution of (11) using the 2-iteration of (FIM) with $h=-0.5$.

The convergence of the proposed (FIM) for Eq.(11) was controlled, via the $h$-curve presented in Figure.1, which is obtained based on the second-order (FIM) approximate solutions. For the non-fractional case both exact results and approximate solutions obtained for the first two approximations are plotted in Figure.2 (a) (resp. Figure.2(b)), they look almost identical. Furthermore, numerical values of the solution in the case $\alpha=1$ are tabulated and compared, in table.1, with the exact solutions and those obtained by Molliq et al. [8] using (VIM), it is to be noted that (FIM) provides accurate solutions only in 2-iterations compared to those found by (VIM) in 3-iterations, which demonstrates the efficiency of (FIM). 
Table 1: Comparison of the FIM solutions of (11) with the exact and the VIM solutions for $\alpha=1$ and $\rho=0.001$

\begin{tabular}{cccccc}
\hline$x$ & $y$ & $t$ & FIM sol.(2-iterations) & VIM sol.(3-iterations [8]) & Exact sol. \\
\hline 0.1 & 0.1 & 0.2 & $5.390593222 .10^{-5}$ & $5.355355975 .10^{-5}$ & $5.393877159 .10^{-5}$ \\
& & 0.3 & $5.381610466 .10^{-5}$ & $5.330816448 .10^{-5}$ & $5.388407669 .10^{-5}$ \\
& & 0.4 & $5.371382992 .10^{-5}$ & $5.306406852 .10^{-5}$ & $5.382941057 .10^{-5}$ \\
0.6 & 0.6 & 0.2 & $3.003983112 .10^{-3}$ & $2.989813669 .10^{-3}$ & $3.036507411 .10^{-3}$ \\
& & 0.3 & $2.987140698 .10^{-3}$ & $2.967173317 .10^{-3}$ & $3.035778955 .10^{-3}$ \\
& & 0.4 & $2.970394131 .10^{-3}$ & $2.945226366 .10^{-3}$ & $3.035050641 .10^{-3}$ \\
0.9 & 0.9 & 0.2 & $1.113620846 .10^{-2}$ & $1.102484681 .10^{-2}$ & $1.153697757 .10^{-2}$ \\
& & 0.3 & $1.094772007 .10^{-2}$ & $1.079635470 .10^{-2}$ & $1.153454074 .10^{-2}$ \\
& & 0.4 & $1.076839361 .10^{-2}$ & $1.057416210 .10^{-2}$ & $1.153210438 .10^{-2}$ \\
\hline
\end{tabular}
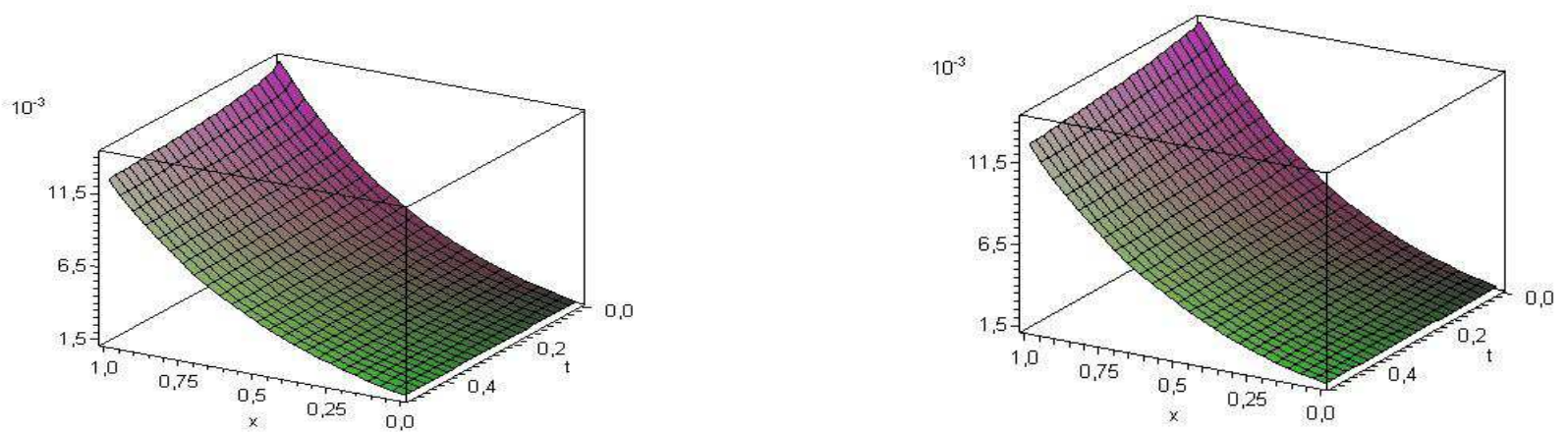

(a)

Figure 3: Profiles of $u(x, y, t)$ solution of (11) using the 2-iteration of (FIM) with $\rho=0.001, h=-0.5$ and $y=0.9$ : (a) $\alpha=0.5$ and (b) $\alpha=0.75$.

Table 2: Numerical solutions for (11) using 2-iterations and $h=-0.5$, when $\alpha=0.5, \alpha=0.75$ and $\rho=0.001$

\begin{tabular}{ccccc}
\hline$x$ & $y$ & $t$ & $\alpha=0.5$ & $\alpha=0.75$ \\
\hline 0.1 & 0.1 & 0.2 & $5.350303782 .10^{-5}$ & $5.377345226 .10^{-5}$ \\
& & 0.3 & $5.331197719 .10^{-5}$ & $5.363544622 .10^{-5}$ \\
& & 0.4 & $5.313136488 .10^{-5}$ & $5.349006821 .10^{-5}$ \\
0.6 & 0.6 & 0.2 & $2.953670347 .10^{-3}$ & $2.983018422 .10^{-3}$ \\
& & 0.3 & $2.935230699 .10^{-3}$ & $2.963801805 .10^{-3}$ \\
& & 0.4 & $2.919818329 .10^{-5}$ & $2.946290643 .10^{-3}$ \\
0.9 & 0.9 & 0.2 & $1.065695617 .10^{-2}$ & $1.091496633 .10^{-2}$ \\
& & 0.3 & $1.050564728 .10^{-2}$ & $1.072196895 .10^{-2}$ \\
& & 0.4 & $1.039024574 .10^{-2}$ & $1.055758009 .10^{-2}$ \\
\hline
\end{tabular}

\section{Conclusion}

In this paper, the fractional iteration method (FIM) has been successfully applied to study a time-fractional Zakharov-Kuznetsov equation. The results show that fractional iteration method is an efficient and easy-to-use technique for finding exact and approximate solutions for nonlinear fractional partial differential equations. On the other hand, the method was used in a direct way without need for the Lagrange multiplier, correction functional, stationary conditions, linearization or discretization. 


\section{References}

[1] F. Abidi, K. Omrani: The homotopy analysis method for solving the Fornberg-Whitham equation and comparison with Adomian's decomposition method, Comput. Math. Appl. 59 (2010) 2743-2750.

[2] J.H. He: Approximate analytical solution for seepage flow with fractional derivatives in porous media, Comput. Methods Appl. Mech. Engrg. 167 (1998) pp.57-68.

[3] A. Ghorbani: Toward a new analytical method for solving nonlinear fractional differential equations, Comput. Methods Appl. Mech. Engrg. 197 (2008) pp.4173-4179.

[4] M.Shateri and D.D.Ganji: Solitary Wave Solutions for a time-fraction generalized Hirota-Satsuma coupled KdV equation by a new analytical technique, Int.J.Diff.Eqs., 11 pages doi:10.1155/2010/954674.

[5] V.E Zakharov and E.A. Kuznetsov: On three-dimensional solitons, Sov. Phys. JETP 39 (1974), pp.285-286.

[6] S.Munro and E.J.Parkes: The derivation of a modified Zakharov-Kuznetsov equation and the stability of its solutions. J. of Plasma Phy. 64 (1999), pp.305-317.

[7] K. Batiha: Approximate analytical solution for the Zakharov-Kuznetsov equations with fully nonlinear dispersion, J. Comput. Appl. Math. 216 (2009), pp.157-163.

[8] R.Y.Molliq, M.S.M. Noorani, I. Hashim and R.R. Ahmad: Approximate solutions of fractional Zakharov-Kuznetsov, J. of Comput. and Appl. Maths 233 (2009) pp.103-108. equations by VIM

[9] A.Yildirim and Y.Gulkanat : Analytical Approach to Fractional Zakharov-Kuznetsov Equations by He's Homotopy Perturbation Method, Commun. Theor. Phys. 53 (2010), pp.1005-1010.

[10] I. Podlubny, Fractional Differential Equations, Academic Press, San Diego, CA, 1999.

[11] M. Caputo, Linear models of dissipation whose Q is almost frequency independent, Part II, Geophys. J. R. Astr. Soc. 13 (1967), 529-539.

[12] M. Reed and B. Simon, Methods of Modern Mathematical Physics, I: Functional Analysis, Academic Press, New York, NY, USA, 1980.

[13] S.J. Liao: On the homotopy analysis method for nonlinear problems, Appl. Math. Comput. 147 (2004), pp.499-513.

[14] M. Inc, Exact solutions with solitary patterns for the Zakharov-Kuznetsov equations with fully nonlinear dispersion, Chaos Solitons Fractals 33 (2007) pp.1783-1790.

[15] S. Abbasbandy, E. Shivanian and K. Vajravelu: Mathematical properties of $h$-curve in the frame work of the homotopy analysis method. Comm. in Nonl. Sci. and Num. Sim., In press, (2011).

[16] Yildiray Keskin, Onur Karaoglu, Sema Servi and Galip Oturanc, The approximate solution of high-order linear fractional differential equations with variable coefficients in terms of generalized Taylor polynomials, Mathematical and Computational Applications, Vol. 16, No. 3, pp. 617-629, 2011. 